\title{
The Effect of Yaw Angle on a Compressible Rectangular Cavity Flow
}

\author{
Kuan-Huang Lee, ${ }^{1}$ Kung-Ming Chung, ${ }^{2}$ and Keh-Chin Chang ${ }^{1}$ \\ ${ }^{1}$ Institute of Aeronautics and Astronautics, National Cheng Kung University, Tainan, Taiwan \\ ${ }^{2}$ Aerospace Science and Technology Research Centre, National Cheng Kung University, Tainan, Taiwan \\ Correspondence should be addressed to Kung-Ming Chung; kmchung@mail.ncku.edu.tw
}

Received 6 December 2016; Accepted 10 April 2017; Published 24 April 2017

Academic Editor: Saad A. Ahmed

Copyright ( 2017 Kuan-Huang Lee et al. This is an open access article distributed under the Creative Commons Attribution License, which permits unrestricted use, distribution, and reproduction in any medium, provided the original work is properly cited.

\begin{abstract}
Experiments are performed to determine the characteristics of a compressible flow over yawed rectangular cavities for Mach numbers of $0.64,0.70$, and 0.83 . The cavity's length-to-depth ratio varies from 4.43 to 21.50 and the length-to-width ratio is unity. The yaw angle is $0^{\circ}-45^{\circ}$. The upstream compression and downstream expansion near the front and rear corners of a cavity decrease when the value of the yaw angle increases. The amplitude of the fluctuating pressure is a maximum for an open cavity with a yaw angle of $15^{\circ}$. An increase in the yaw angle results in a reduction in the pressure fluctuations for both open and transitional cavities. In the span-wise direction, variations in the mean and fluctuating pressure are less significant than those in the chord-wise direction. The oscillating frequency of resonance varies slightly with the yaw angle, but the amplitudes for the power spectral density are significantly reduced when the yaw angle is larger than $30^{\circ}$. For lower Mach numbers, the lower mode plays an important role in self-sustained oscillations for an open cavity when there is an increase in the yaw angle.
\end{abstract}

\section{Introduction}

The presence of a cavity changes the mean and fluctuating pressure distributions inside and near a cavity $[1,2]$. For compressible flow in a rectangular cavity $(M=0.2-0.95)$, the mean and fluctuation pressure distributions normal to the direction of the flow depend principally on the length-todepth ratio, $L / H$ [3-5]. When $L / H>13$ (a closed cavity), the flow expands from the leading edge, attaches to the floor, and separates ahead of the rear face of the cavity. This results in a significant variation in the mean surface pressure in the stream-wise direction. The shear layer for an open cavity $(L / H<6-8)$ spans the cavity and impinges near the rear corner. Discrete acoustic tones are associated with a feedback loop between vortex shedding and acoustic disturbance, which is known as Rossiter resonance [6,7].

For flow in a yawed rectangular cavity, the yaw angle, $\beta$, is defined as the angle between the freestream and the chordwise direction of the cavity. An asymmetric flow pattern inside the cavity can be expected. Savory et al. $\left(U_{\infty}=\right.$ $7 \mathrm{~m} / \mathrm{s}$ ) [8] measured the drag of flow in a yawed cavity for
$L / H=1.428-10.0$ and $\beta=0^{\circ}-90^{\circ}$, in which the maximum drag occurs for $L / H=2-2.5$ and for $\beta=45-60 \mathrm{deg}$. They also noted that the drag for a square cavity (width-to-length, $W / L$ $=1$ ) is greater than that for a rectangular cavity with the same plan-form area. Czech et al. $\left(U_{\infty}=16 \mathrm{~m} / \mathrm{s}\right)$ [9] demonstrated a critical value of $\beta$ of $45^{\circ}$, based on the measurements of mean and fluctuating pressure in a wide cavity $(W / L=$ 4.85 ), and showed that asymmetry is more apparent for a deep cavity $(L / H=1-3)$. The oil flow visualization and drag measurements by Gai et al. $\left(U_{\infty}=15 \mathrm{~m} / \mathrm{s}\right)[10]$ showed that the most asymmetric flow pattern inside a cavity and the lowest drag occur at $\beta=45^{\circ}$ for $L / H=6-16$.

In terms of self-sustained oscillations, Bari and Chambers $\left(U_{\infty}=20-44 \mathrm{~m} / \mathrm{s}\right)[11]$ showed that the yaw angle for a cavity need not significantly affect the resonant frequencies. However, there may be a switch in the dominant mode and the effective stream-wise length, $L / \cos (\beta)$, of a cavity at yaw is probably not a suitable characteristic length. A study by Lee et al. $\left(L / H=5.0, \beta=0^{\circ}-20^{\circ}\right)[12]$ showed that the resonance switches from 2 nd mode to 3 rd mode at $\beta=15^{\circ}$ for transonic and low supersonic flows ( $M=0.84$ and 1.10). The strength of 

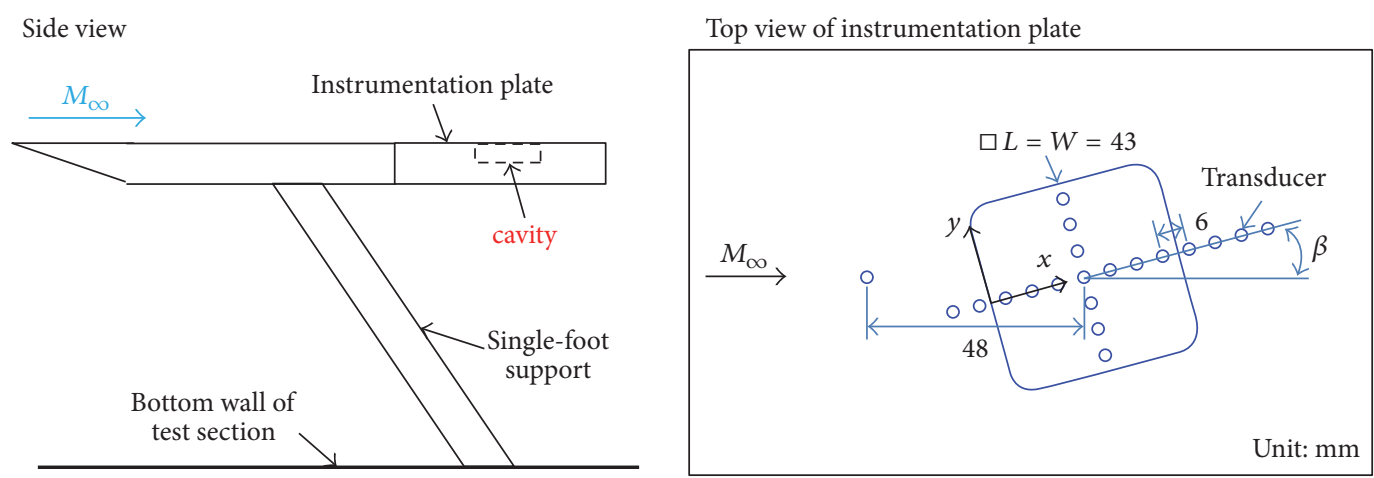

FIgURE 1: Test configuration.

the resonance is also significantly affected by the value of $\beta$. The overall fluctuation is increased at a critical yaw angle for a subsonic flow $\left(U_{\infty}=25 \mathrm{~m} / \mathrm{s}\right)$ [13] and significantly reduced for a supersonic flow $(M=2.0)$ [14].

Since less work has been done for a compressible turbulent flow past rectangular cavities at yaw in the past, this experimental study aims to characterize the flow in detail. The chord-wise and span-wise distributions of the mean and fluctuating pressure are determined. The distribution of the power spectral density near the rear face is used to characterize self-sustained oscillations for both open and transitional cavity flows. The resonant frequencies are calculated and the corresponding Strouhal numbers were compared with those predicted using Rossiter's semiempirical formula, in which the empirical parameters are determined using a gradientbased searching method.

\section{Experimental Techniques}

2.1. The Transonic Wind Tunnel and Instrumentation. The transonic wind tunnel at the Aerospace Science and Technology Research Center in National Cheng Kung University is a blow-down type. The test section for this study had solid side walls and perforated top and bottom walls. It was $600 \mathrm{~mm}$ square and $1500 \mathrm{~mm}$ in length. Chung et al. [15] showed that perforated walls induce strong acoustic waves, for which the characteristic frequency is $4.2-4.8 \mathrm{kHz}$ for $M$ $=0.64-0.83$. The stagnation pressure was controlled using a rotary perforated sleeve valve and, for subsonic flow, the test Mach number, $M$, was monitored using two choked flaps. The stagnation pressure and temperature were, respectively, $172 \pm 1 \mathrm{kPa}(25.0 \pm 0.15 \mathrm{psia})$ and room temperature, for $M=$ $0.64,0.70$, and $0.83 \pm 0.01$.

A National Instruments (NI-SCXI) system recorded the output signals from the dynamic pressure transducers (Kulite XCS-093-25A, B screen). The natural frequency of the transducers is $200 \mathrm{kHz}$, as quoted by the manufacturer. The transducers were powered by a DC power supply of $10.0 \mathrm{~V}$ (GW Instek PSS-3203) and Ectron amplifiers (753A), which had a roll-off frequency of approximately $140 \mathrm{kHz}$ at a gain of
20 , were used to improve the signal-to-noise ratio. The sample time was $5 \mu$ s and each sample record contained 131,072 data points. Each sample record was then divided into 32 subsets of 4096 data points for data analysis. The experimental results for the flat plate case show that the respective uncertainty in the values for the static pressure coefficient, $C_{p}$, and the surface fluctuating pressure coefficient, $C_{\sigma_{p}}$, is $2.4 \%$ and $0.4 \%$. Each sample record was then divided into 31 segments with a $50 \%$ overlap and the corresponding frequency resolution was $24.4 \mathrm{~Hz}$ for each segment of 8192 data points. The power spectral density (PSD) was evaluated using a Hann window and a fast Fourier transform. Each spectrum was then generated by averaging 31 spectra for each test case. A factor of $8 / 3$ for each spectrum was used to compensate for the loss that results from the Hann window [16].

2.2. Models and Test Conditions. The test model consisted of a flat plate $(150 \mathrm{~mm} \times 450 \mathrm{~mm})$ that naturally develops a turbulent boundary layer and an instrumentation plate (150 mm square) with a yawed rectangular cavity, as shown in Figure 1. The pressure transducers were flush-mounted along the centerline of each cavity in the chord-wise $(y / L=$ $0)$ and span-wise $(x / L=0.5)$ directions. The distance between the leading edges of the flat plate and the cavity's leading edge was approximately $480 \mathrm{~mm}$. The origin of the Cartesian coordinates was set at the center of the leading edge of the cavity. The positive direction of the $x$-axis is in the chordwise direction towards the trailing edge. The boundary layer thickness was approximately $7 \mathrm{~mm}$, upstream of the cavity's leading edge [4]. The unit Reynolds numbers were 12.9-17.2 $\times$ $10^{6}$ per meter for $M=0.64-0.83$. The geometry of the cavities is summarized in Table 1, where 23 instrumentation plates were fabricated. For a fixed length $(L=43 \mathrm{~mm})$ with different depths $(H=2.0-9.7 \mathrm{~mm})$, the value for $L / H$ ranges from 4.43 to 21.50 and $\beta=5^{\circ}, 10^{\circ}, 15^{\circ}, 30^{\circ}$, and $45^{\circ}$. The data for a rectangular cavity that is normal to the flow direction $\left(\beta=0^{\circ}\right)$ that was gathered by Chung [17] is also included for comparison. Notably, the self-sustained oscillation corresponds to open and transitional-open cavities, for which the value of $L / H=$ 4.43-8.60 ( $H=5.0-9.7 \mathrm{~mm})[4]$. 

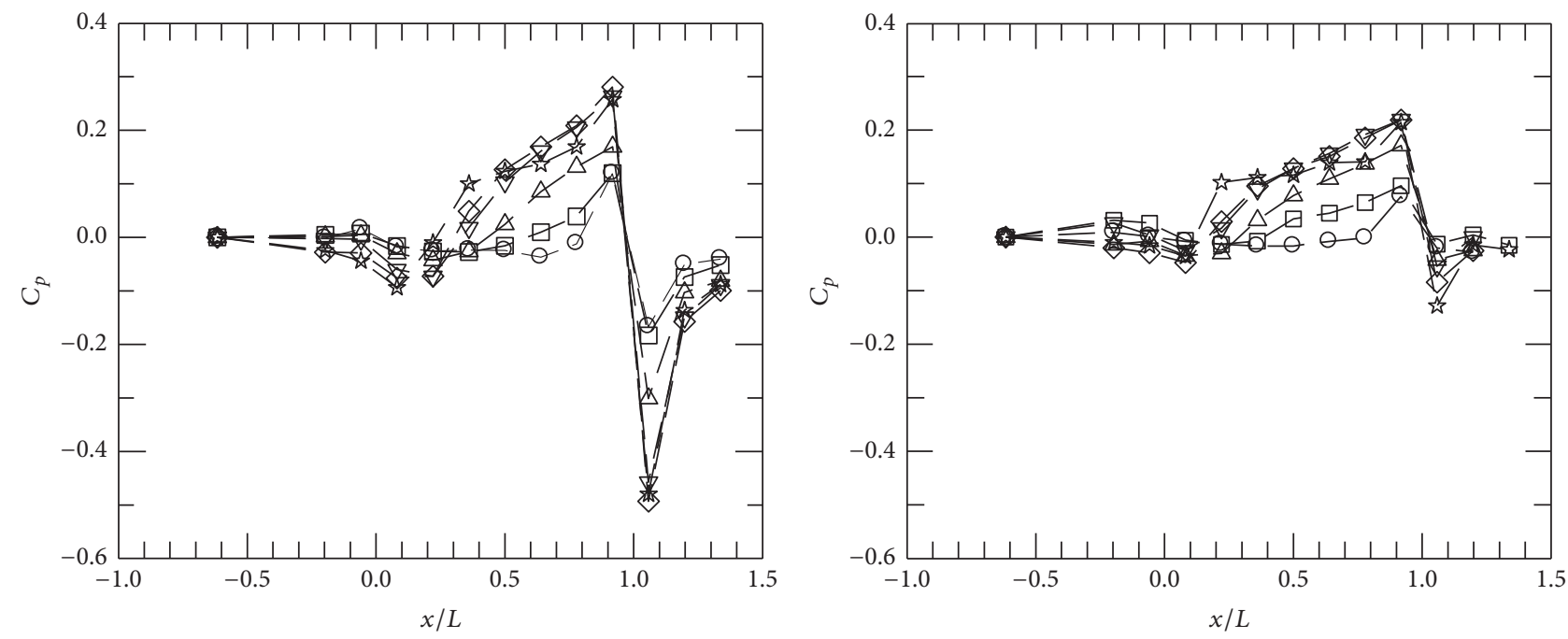

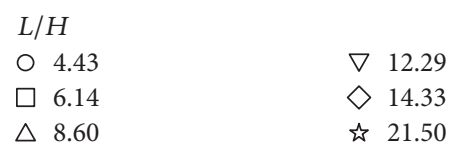

(a) $\beta=10^{\circ}$

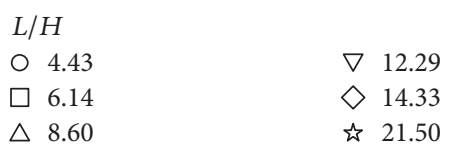

(b) $\beta=45^{\circ}$

Figure 2: The chord-wise distributions of the mean surface pressure at $M=0.83$ : (a) $\beta=10^{\circ}$ and (b) $\beta=45^{\circ}$.

TABLE 1: The geometry of the yawed rectangular cavities.

\begin{tabular}{lcccccc}
\hline$\beta$, deg. & & & $H, \mathrm{~mm}$ & & \\
& 2.0 & 3.0 & 3.5 & 4.0 & 5.0 & 7.0 \\
\hline 5 & $\vee$ & $/$ & $/$ & $/$ & $\vee$ & $\vee$ \\
10 & $\vee$ & $\vee$ & $/$ & $/$ & $\vee$ & $\vee$ \\
15 & $\vee$ & $/$ & $/$ & $/$ & $\vee$ & $\vee$ \\
30 & $\vee$ & $/$ & $/$ & $\vee$ & $\vee$ & $\vee$ \\
45 & $\vee$ & $/$ & $\vee$ & $\vee$ & $\vee$ \\
\hline
\end{tabular}

2.3. Empirical Constants in Rossiter's Formula. A semiempirical formula for a rectangular cavity flow was derived by Rossiter [6] as follows:

$$
\mathrm{St}_{n}=\frac{f_{n} L}{U_{\infty}}=\frac{n-\alpha}{M+1 / k_{c}} .
$$

The $n$ th-mode Strouhal number, $\mathrm{St}_{n}$, is calculated using the oscillation frequency, $f_{n}$, and the freestream velocity, $U_{\infty} . M$ is the freestream Mach number. The empirical parameter, $\alpha$, corresponds to the lag time between the passage of a vortex and the emission of an acoustic pulse and $k_{c}$ is the ratio of the convection velocity for the vortices to $U_{\infty}$. Using a best fit to the measured data, Rossiter proposed values of $\alpha=$ 0.25 and $k_{c}=0.57$ for rectangular cavities. However, Ünalmis et al. [18] showed that the empirical parameters depend on flow conditions and the value of $L / H$. For a cavity at yaw, the optimal values of the empirical parameters are evaluated by minimizing the difference between the experimental and the predicted Strouhal numbers. The steepest descent optimization algorithm is used [19] in this study and the details are given in [20].

\section{Results and Discussion}

3.1. Surface Mean Pressure Distributions. Examples of the $C_{p}$ distributions for $M=0.83$ are shown in Figure 2. For $L / H=12.29,14.33$, and 21.50 and $\beta=10^{\circ}$ (Figure 2(a)), the flow expands near the leading edge and compresses towards the rear face. A reduction in the value of $C_{p}$ is observed downstream of the rear face, following a recovery process. This corresponds to a transitional-closed cavity or a closed cavity $[3,18]$. For $L / H=8.60$, the expansion near the leading edge of the cavity abates. This is termed as transitional-open cavity. For open cavities $\left(L / H=4.43\right.$ and 6.14), a uniform $C_{p}$ distribution inside the cavity is observed for values of $x / L$ up to $0.5-0.7$, following the formation of an adverse pressure gradient near the rear face. When the value of $\beta$ increases $\left(=45^{\circ}\right.$, Figure $2(\mathrm{~b}))$, the $C_{p}$ distribution exhibits a similar pattern for a given value of $L / H$. However, there is less significant 

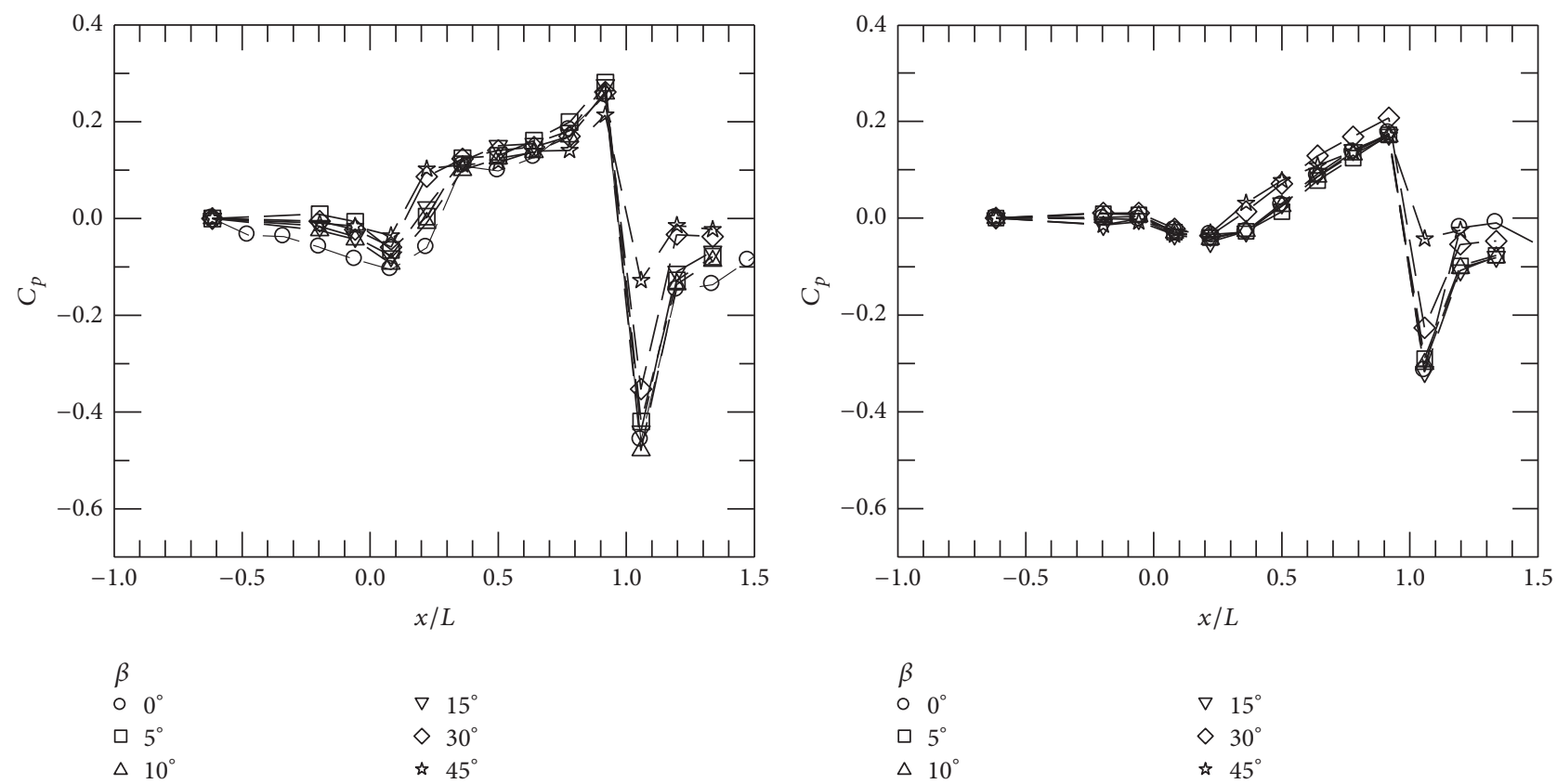

(a) $L / H=21.50$

(b) $L / H=8.60$
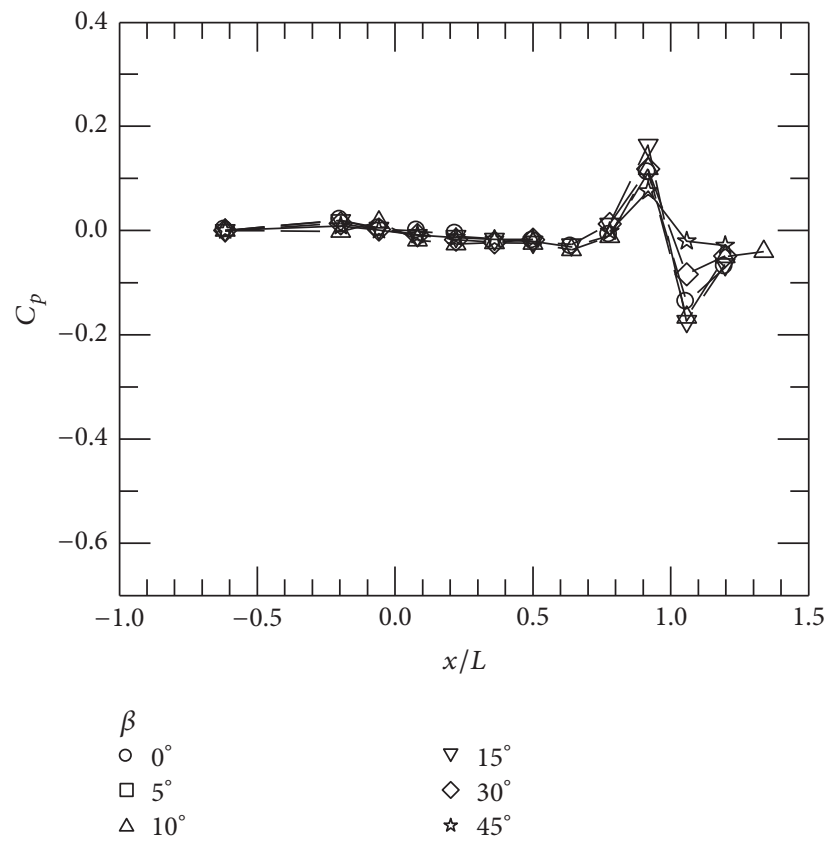

(c) $L / H=4.43$

Figure 3: Chord-wise distributions of the surface mean pressure for $M=0.83$ : (a) $L / H=21.50$, (b) $L / H=8.60$, and (c) $L / H=4.43$.

leading-edge expansion, compression on the cavity floor, and expansion near the rear face. The $C_{p}$ distributions show that the boundaries for the flow type depend more on the value of $L / H$ than on the value of $\beta$.

The effect of the value of $\beta$ on a rectangular cavity flow is shown in Figure 3, where $M=0.83$ and $L / H=4.43,8.60$, and 21.50. For a given value of $L / H$, the $C_{p}$ distributions show that there is less expansion near the rear face when the value of $\beta$ is increased. For $M=0.64$ and 0.70 , the $C_{p}$ distributions show a similar feature. The mean surface pressure near the front and rear face of a cavity is also used to characterize the upstream and downstream influence. For $x / L=-0.058$, the value of $C_{p}$ upstream of the cavities is shown in Figure 4. For a given value of $M$, the value of $\beta$ has a minor effect on the amplitude of $C_{p}$. The Mach number effect is also not significant. Figure 5 shows the variation in $C_{p}$ with the value 

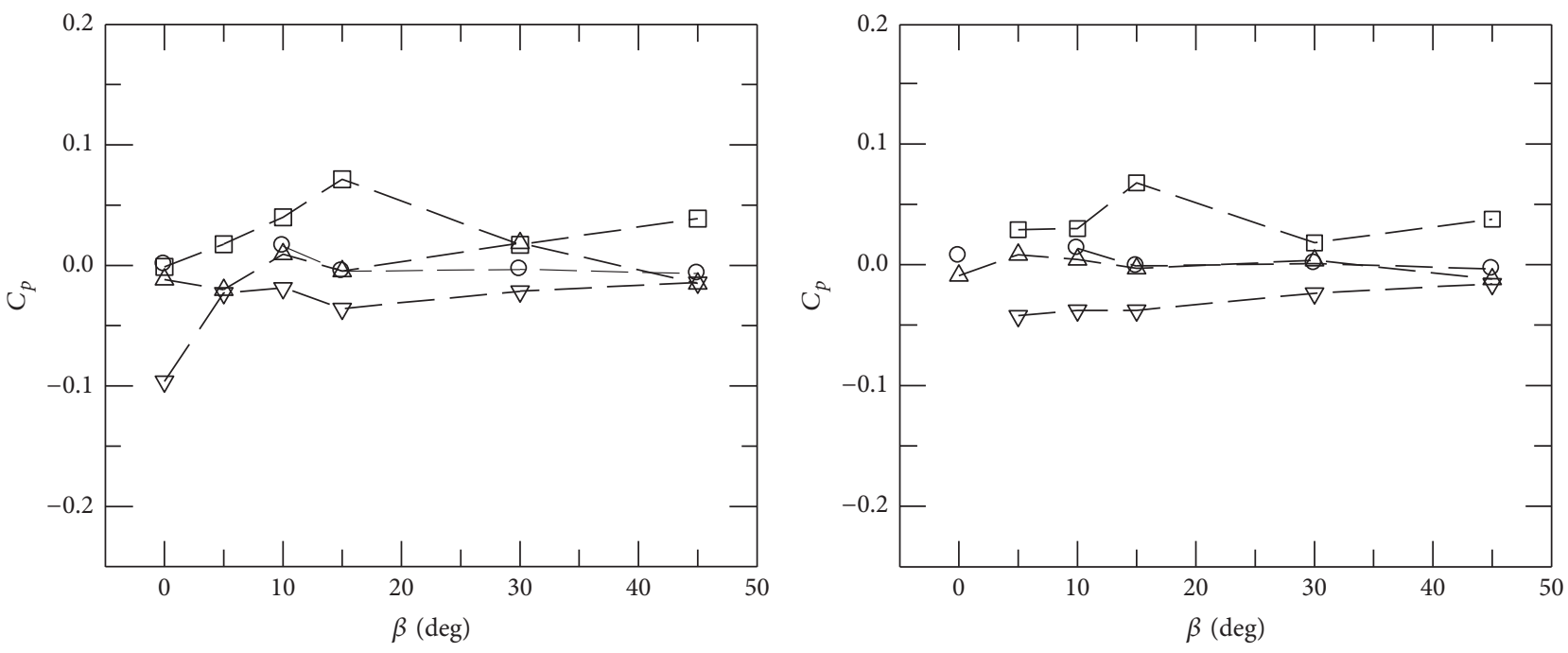

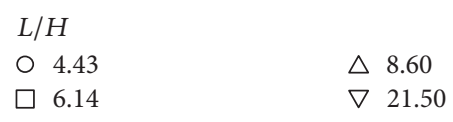

(a) $M=0.64$
$L / H$
○ 4.43
$\triangle 8.60$
$\square 6.14$
$\nabla 21.50$

(b) $M=0.70$

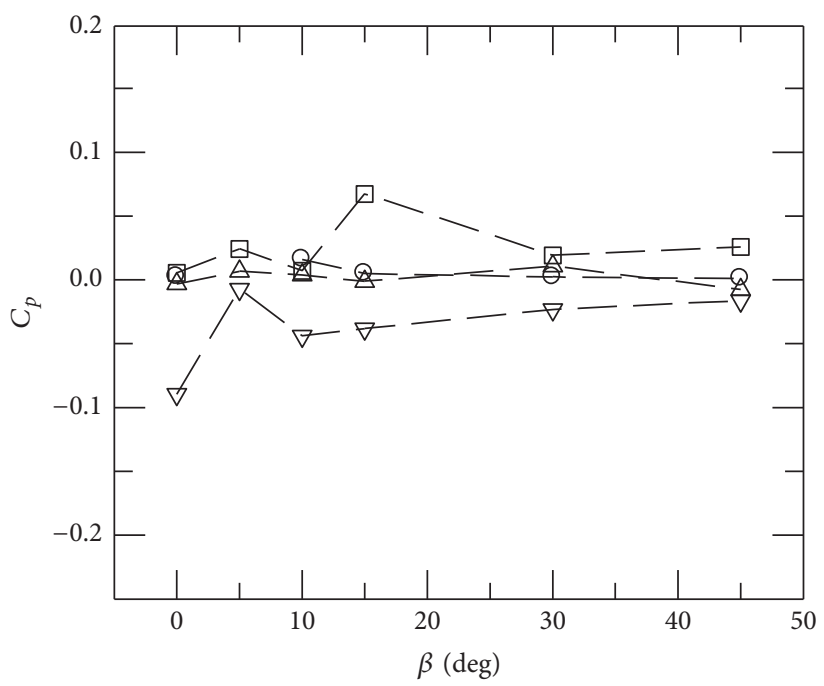

$L / H$

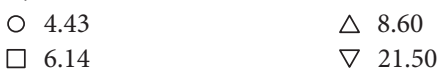

(c) $M=0.83$

FIgURE 4: The pressure coefficient upstream of the cavity $(x / L=-0.058)$ : (a) $M=0.64$, (b) $M=0.70$, and (c) $M=0.83$.

of $\beta$ near the rear face $(x / L=0.919$ and 1.058 , which are, resp., labeled as hollow and solid symbols). The amplitude of $C_{p}$ at $x / L=0.919$ for a closed cavity $(L / H=21.5)$ is greater than that for a transitional-open $(L / H=8.60)$ or an open cavity $(L / H=$ 4.43 and 6.14). The value of $\beta$ has a more significant effect on the amplitude of $C_{p}$ for a value of $x / L=1.058$ than for a value of $x / L=0.919$. There is a minor variation in the amplitude of $C_{p}$ for values of $\beta$ up to $15^{\circ}$, following an increasing $C_{p}$ as the value of $\beta$ increases. For a closed cavity, there is also an increase in the pressure difference for $x / L=0.919$ and 1.058 (greater expansion strength) near the rear face.

3.2. Surface Fluctuating Pressure Distributions. The $C_{\sigma_{p}}$ distributions for $M=0.83$ are shown in Figure 6. At a value of $\beta=$ $10^{\circ}$, the shear layer for an open cavity $(L / H=4.43$ and 6.14$)$ separates from the leading edge of a cavity and impinges near the rear face. The amplitude of $C_{\sigma_{p}}$ increases gradually and reaches a peak value near the rear face. This corresponds to 

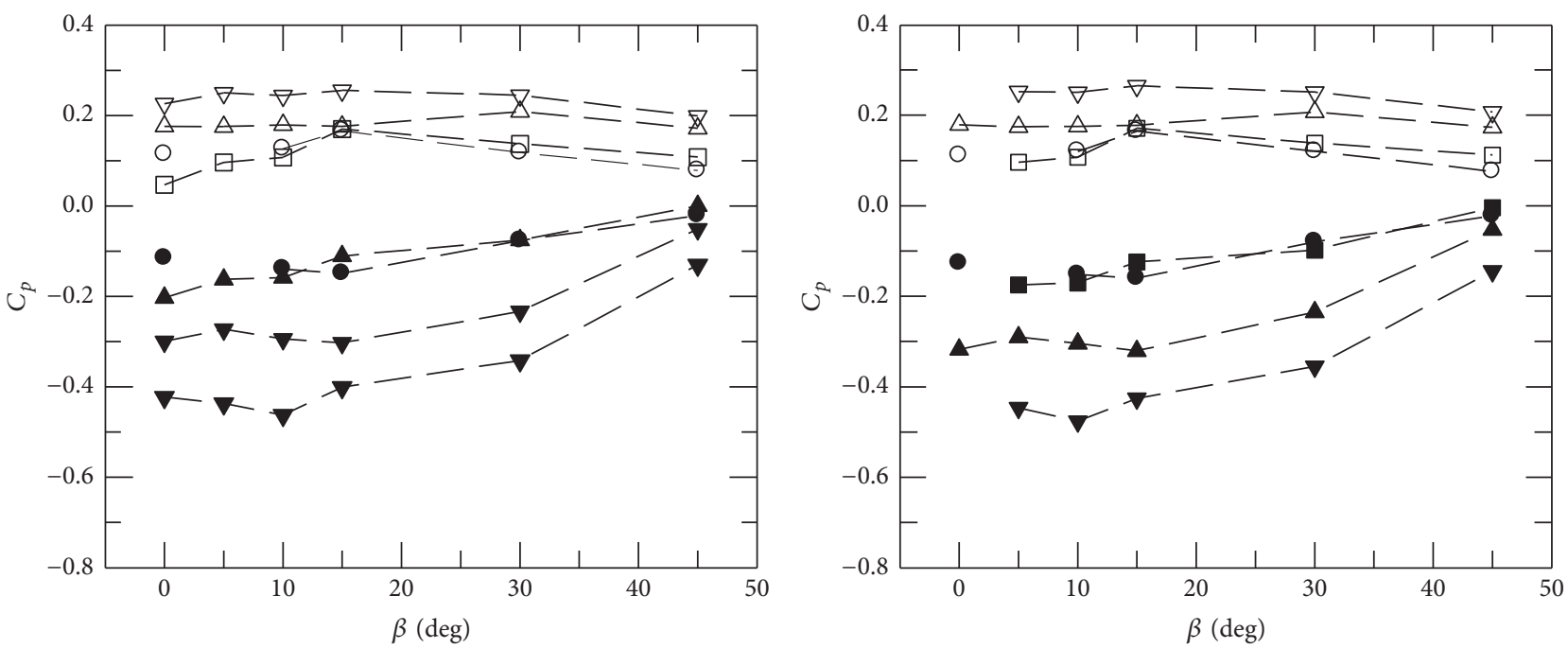

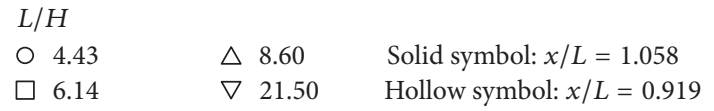

(a) $M=0.64$

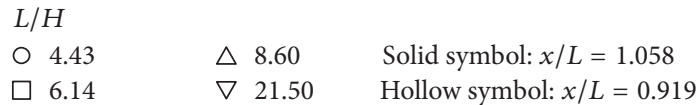

(b) $M=0.70$

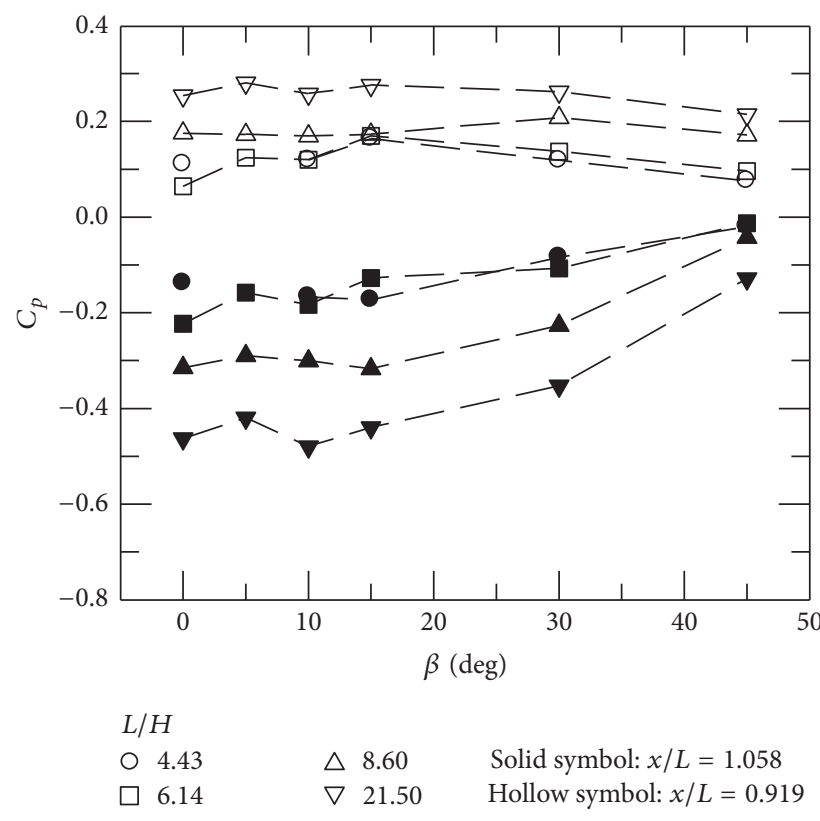

(c) $M=0.83$

FIGURE 5: The pressure coefficients near the rear corner $(x / L=0.919$ and 1.058$)$ : (a) $M=0.64$, (b) $M=0.70$, and (c) $M=0.83$.

self-sustained oscillation [6]. For a transitional cavity $(L / H=$ 8.60-14.33), there is an increase in the fluctuating pressure near the central region of the cavity $(x / L \approx 0.3-0.7)$. For transitional-closed cavity and closed cavities, minor peak pressure fluctuations are observed for a value of $x / L \approx 0.36$, because of the deflection or the reattachment of shear layer. For an open cavity, the amplitude of $C_{\sigma_{p}}$ increases ahead of the rear corner $(x / L=0.919)$ or when there is a decrease in the value of $L / H$. Downstream of the rear face $(x / L=1.058)$, Heller and Bliss [21] showed that the pressure fluctuations are associated with the balance between the energy that is supplied by the external flow and the energy that is dissipated by viscous losses and acoustic radiation. The peak pressure fluctuations, $C_{\sigma_{p, \max }}$, correspond to the unsteady process for the addition and removal of mass for open and transitional cavities $(L / H=4.43-14.33)$. For a value of $\beta=45^{\circ}$, the $C_{\sigma_{p}}$ distributions are similar to those for a value of $\beta=10^{\circ}$. However, for a transitional cavity, the amplitude of $C_{\sigma_{p}}$ at $x / L=0.919$ is greater than that for an open cavity. Minor 

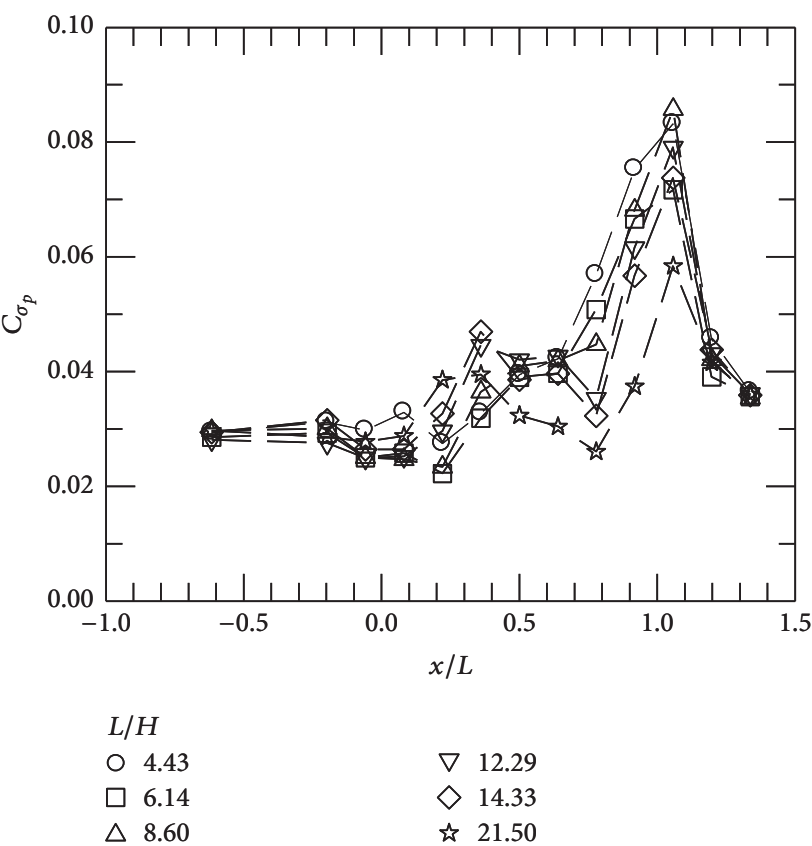

(a) $\beta=10^{\circ}$

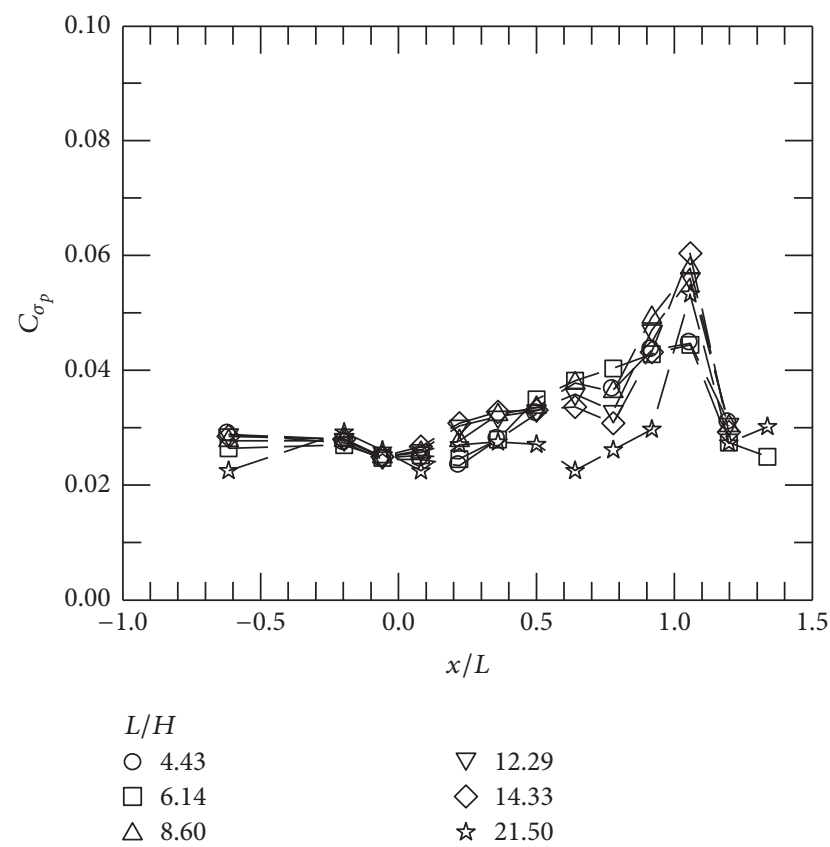

(b) $\beta=45^{\circ}$

Figure 6: Chord-wise distributions of surface fluctuating pressure for $M=0.83$ : (a) $\beta=10^{\circ}$ and (b) $\beta=45^{\circ}$.

peak pressure fluctuations are observed at $x / L=0.64$. The amplitude of $C_{\sigma_{p, \max }}$ at $x / L=1.058$ also decreases significantly when there is an increase in the value of $\beta$.

The effect of the value of $\beta$ on the $C_{\sigma_{p}}$ distributions for $M=0.83$ is shown in Figure 7. For a closed cavity $(L / H=$ 21.50), the minor peak pressure fluctuations for $x / L \approx 0.36$ decrease as the value of $\beta$ increases, as do the peak pressure fluctuations for $x / L=1.058$. For a transitional cavity $(L / H=$ 8.60), the effect of the yaw angle effect is minimal, except when there is a significant reduction in the amplitude of $C_{\sigma_{p}}$ downstream of the rear face for $\beta=45^{\circ}$. For an open cavity $(L / H=4.43)$, the yaw angle has an evident effect on the amplitude of $C_{\sigma_{p}}$ near the rear face. For $M=0.64$ and 0.70, the distributions of $C_{\sigma_{p}}$ are similar to those for $M=0.83$. The effect of $M$ and $\beta$ on $C_{\sigma_{p, \max }}$ are shown in Figure 8. For a given value of $M$, the amplitude of $C_{\sigma_{p, \max }}$ at $\beta=0^{\circ}$ is the greatest for a transitional cavity and the least for a closed cavity. For a closed cavity $(L / H=21.50)$, there is a small variation in the amplitude of $C_{\sigma_{p, \max }}$ as the value of $\beta$ varies and there is a reduction at $\beta=45^{\circ}$ for a transitional cavity $(L / H=8.60)$. For $L / H=4.43$, an increase in the amplitude of $C_{\sigma_{p, \max }}$ is observed up to $\beta=15^{\circ}$, following a decrease as the value of $\beta$ increases. However, the opposite trend is true for $L / H=4.43$ when $\beta=$ $0^{\circ}-15^{\circ}$. It is also seen that, for an open cavity, the amplitude of $C_{\sigma_{p, \max }}$ at $\beta=45^{\circ}$ is less than that for a closed cavity. This demonstrates that the peak pressure fluctuations at $\beta=45^{\circ}$ mainly correspond to the unsteady process for the addition and removal of mass near the rear face and the self-sustained oscillation for an open cavity is attenuated.
3.3. Span-Wise Mean and Fluctuating Pressure Distributions. The mean and fluctuating pressure distributions in the span-wise direction are of interest. Figure 9 shows the $C_{p}$ distributions for $M=0.83$ at $\beta=10^{\circ}$ and $45^{\circ}$. For $x / L=0.5$ and $\beta=10^{\circ}$, the $C_{p}$ distributions for closed and transitionalclosed cavities $(L / H=21.50-12.29)$ show small variations and transitional-open and open cavities $(L / H=8.60-4.43)$ show a slight increase from $y / L=-0.42$ to 0.42 . For a value of $\beta=45^{\circ}$, the $C_{p}$ distributions are asymmetric. This asymmetric feature is more significant for a transitional cavity $(L / H=8.60-14.33)$ and is less evident for closed and open cavities. The amplitude of $C_{p}$ for open and transitional cavities increases when the value of $\beta$ increases, which agrees with results that are shown in Figure 2(b) for $x / L=0.5$. The span-wise fluctuating pressure distributions for $M=0.83$ are shown in Figure 10, which shows a similar feature to that in Figure 6 for $x / L$ $=0.5$. The amplitude of $C_{\sigma_{p}}$ is the least for a closed cavity $(L / H=21.50)$. There are small variations at $\beta=10^{\circ}$ and a gradual decrease from $y / L=-0.42$ to 0.42 at $\beta=45^{\circ}$. Notably, for a given value of $L / H$, the effect of the yaw angle on the amplitude of $C_{p}$ and $C_{\sigma_{p}}$ is more evident near the sidewalls of the cavity $(y / L= \pm 0.42)$, particularly for the values of $\beta=30^{\circ}$ and $45^{\circ}$.

3.4. Power Spectra. Self-sustained oscillation in a cavity is a consequence of periodic vortex shedding and acoustic disturbance. The power spectral density (PSD) for $M=0.83$ at $x / L=0.919$ is shown in Figure 11. The plots are presented in terms of sound pressure level (SPL $=20 \log _{10}\left(p / p_{s}\right), p_{s}=2 \times$ $10^{-5} \mathrm{~Pa}$ ) and are consecutively offset by $10 \mathrm{~dB}$ for clarity. The 

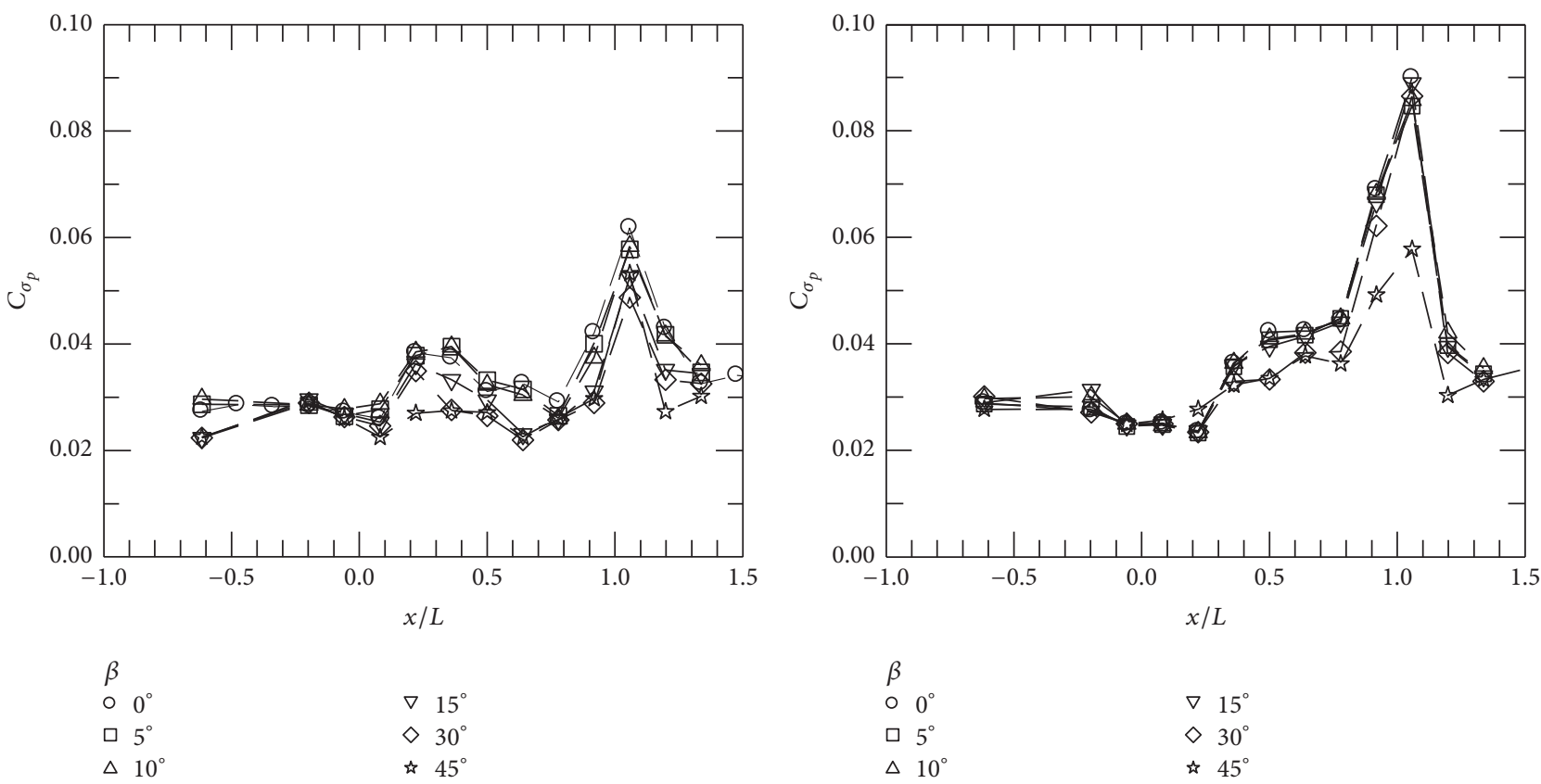

(a) $L / H=21.50$

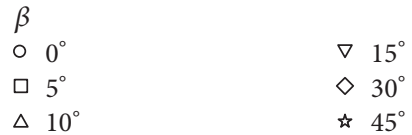

(b) $L / H=8.60$
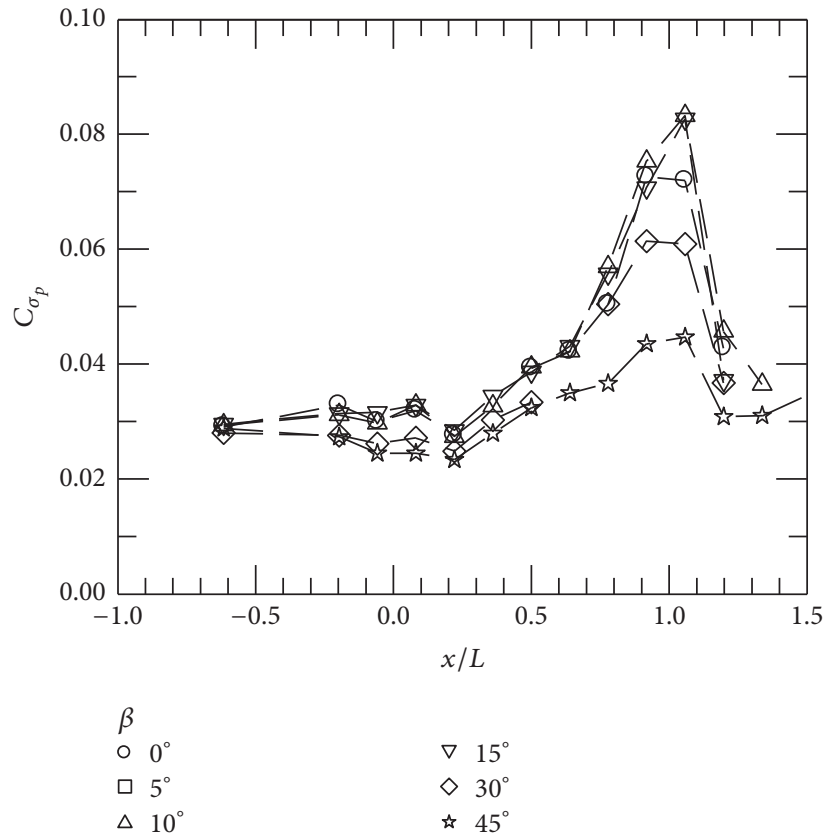

(c) $L / H=4.43$

Figure 7: Chord-wise distributions of the surface fluctuating pressure for $M=0.83$ : (a) $L / H=21.50$, (b) $L / H=8.60$, and (c) $L / H=4.43$.

uppermost plot has its original values. The PSD for a flat plate (FP) flow without the presence of a cavity is also shown, for reference. The peak frequency for this flow is approximately $4600 \mathrm{~Hz}$ and is induced by the perforated wall of the wind tunnel. For open cavities $\left(L / H=4.43\right.$ and 6.14) at $\beta=0^{\circ}$, discrete acoustic tones are observed at $f_{1} \approx 1800, f_{2} \approx 4100$, and $f_{3} \approx 6300 \mathrm{~Hz}$. The values for the SPL for $L / H=4.43$ are larger than those for $L / H=6.14$. The 1st mode is not observed for a transitional-open cavity $(L / H=8.60)$ and the 2nd mode is less apparent. Taking the effect of the yaw angle into account, the frequency of the 1st mode for $L / H=4.43$ and for $\beta=10^{\circ}$ and $15^{\circ}$, as shown in Figure 11(a), is slightly greater than that for $\beta=0^{\circ}$ and the values for the SPL decrease as the value of $\beta$ increases. For the 2 nd and 3 rd modes, an increase in the value of $\beta$ results in a reduction in their frequencies and their amplitudes. Only the 1st mode is evident for $\beta=$ $30^{\circ}$ and self-sustained oscillations are only just evident for $\beta=45^{\circ}$. Figure $11(\mathrm{~b})$ shows that, for $L / H=6.14$, there are 


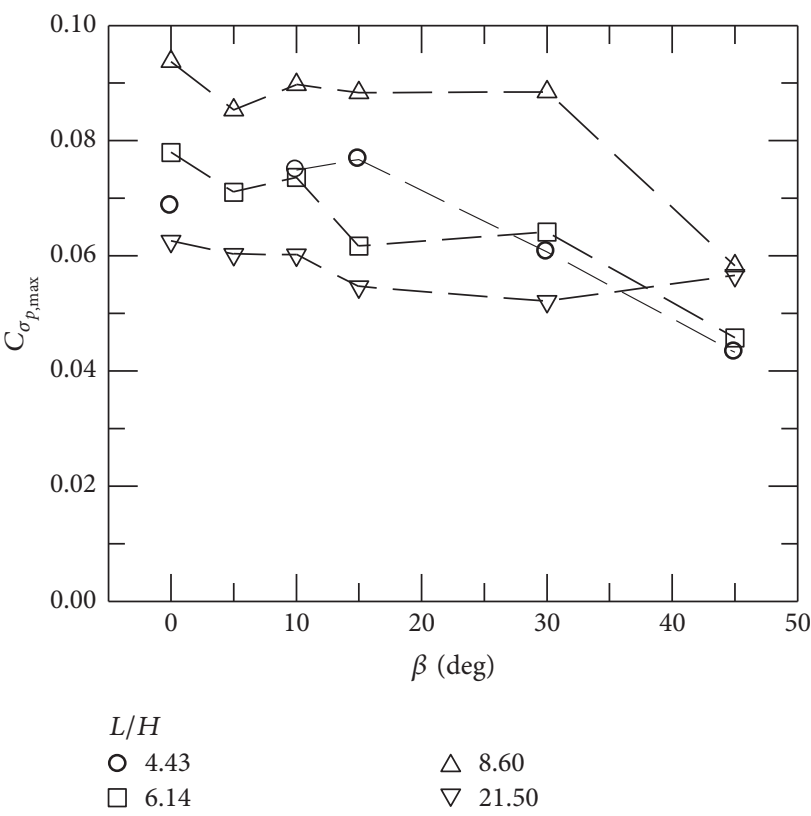

(a) $M=0.64$

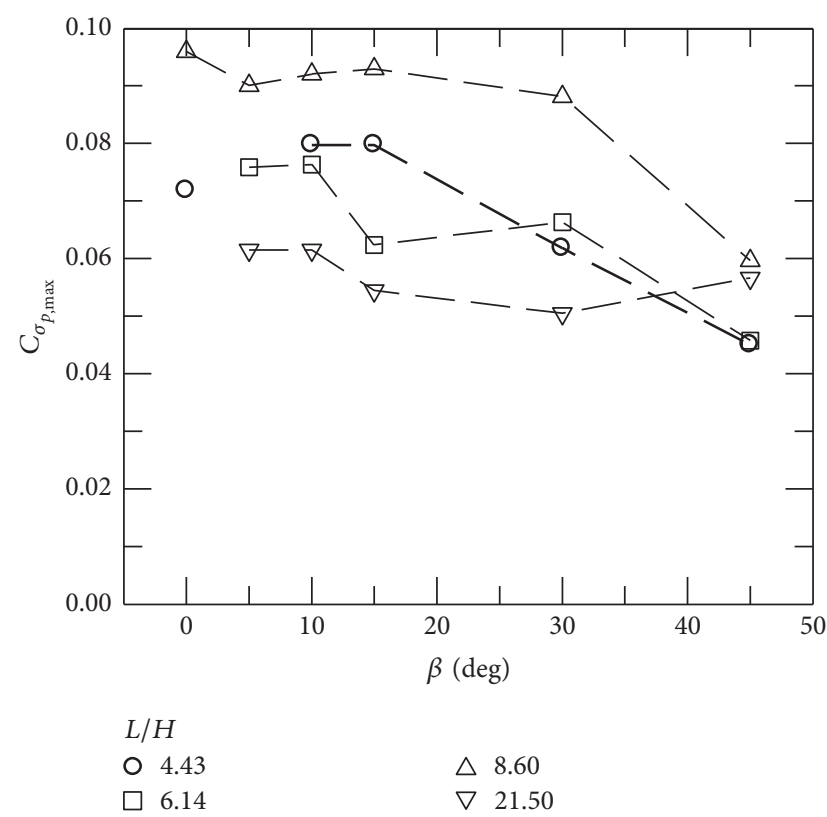

(b) $M=0.70$
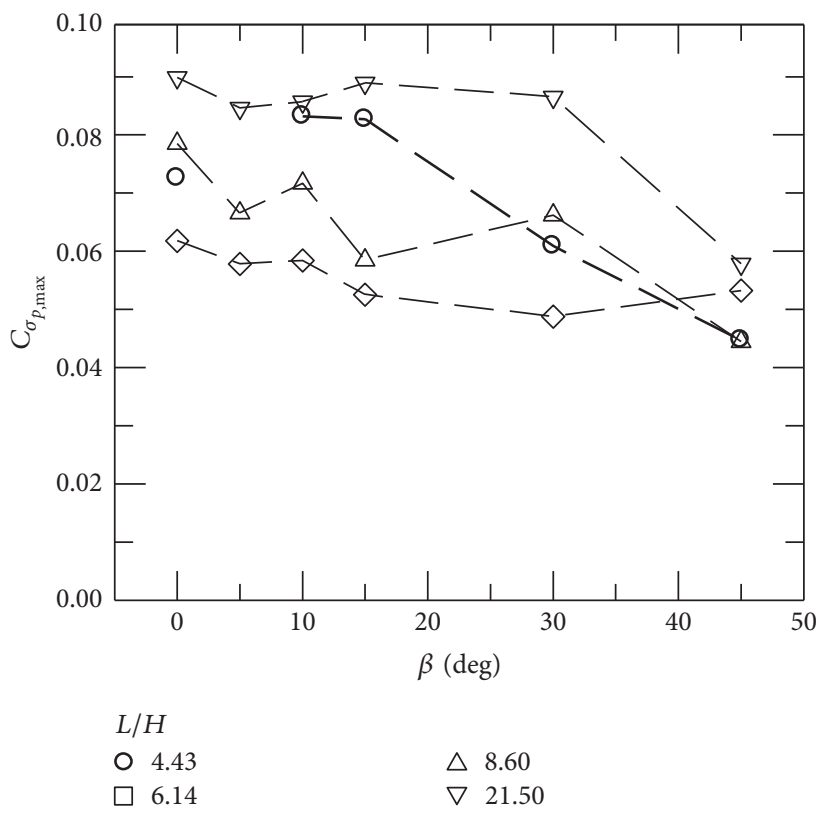

(c) $M=0.83$

FIGURE 8: Peak pressure fluctuations: (a) $M=0.64$, (b) $M=0.70$, and (c) $M=0.83$.

weaker oscillations than for $L / H=4.43$. The 1st mode almost disappears at $\beta=15^{\circ}$ and no modes are visible for $\beta=30^{\circ}$ or $45^{\circ}$. For a transitional-open cavity $(L / H=8.60)$, Figure $11(\mathrm{c})$ shows that only the 2 nd mode is evident for $\beta=5^{\circ}-15^{\circ}$. In summary, the 2 nd mode dominates self-sustained oscillations for a cavity at yaw.

3.5. Self-Sustained Oscillations for a Cavity at Yaw. Previous studies have shown that, for rectangular cavity flow, selfsustained oscillations can be predicted using the semiempirical Rossiter's formula [22]. Figure 12 shows the amplitudes of the resonance for rectangular cavities at yaw, including open $(L / H=4.43$ and 6.14) and transitional-open cavities $(L / H=$ $8.60)$. In general, there is a decrease in the value of SPL as the value of $\beta$ increases and the 2 nd mode dominates. These values for the SPL are greater than those for the 1st and 3rd modes. However, for $M=0.64$, the value for the SPL for the 1st mode is greater than that for the 2nd mode for $L / H=4.43$ and 6.14 , at $\beta=15^{\circ}$ and $30^{\circ}$, and for $M=0.70$ and $0.83(L / H=$ $4.43)$ at $\beta=30^{\circ}$. This shows that the dominant mode changes for different values of $M$ and $\beta$. For $M=0.83$ and $L / H=8.60$, all three modes disappear at $\beta=30^{\circ}$. It is also noted that an 

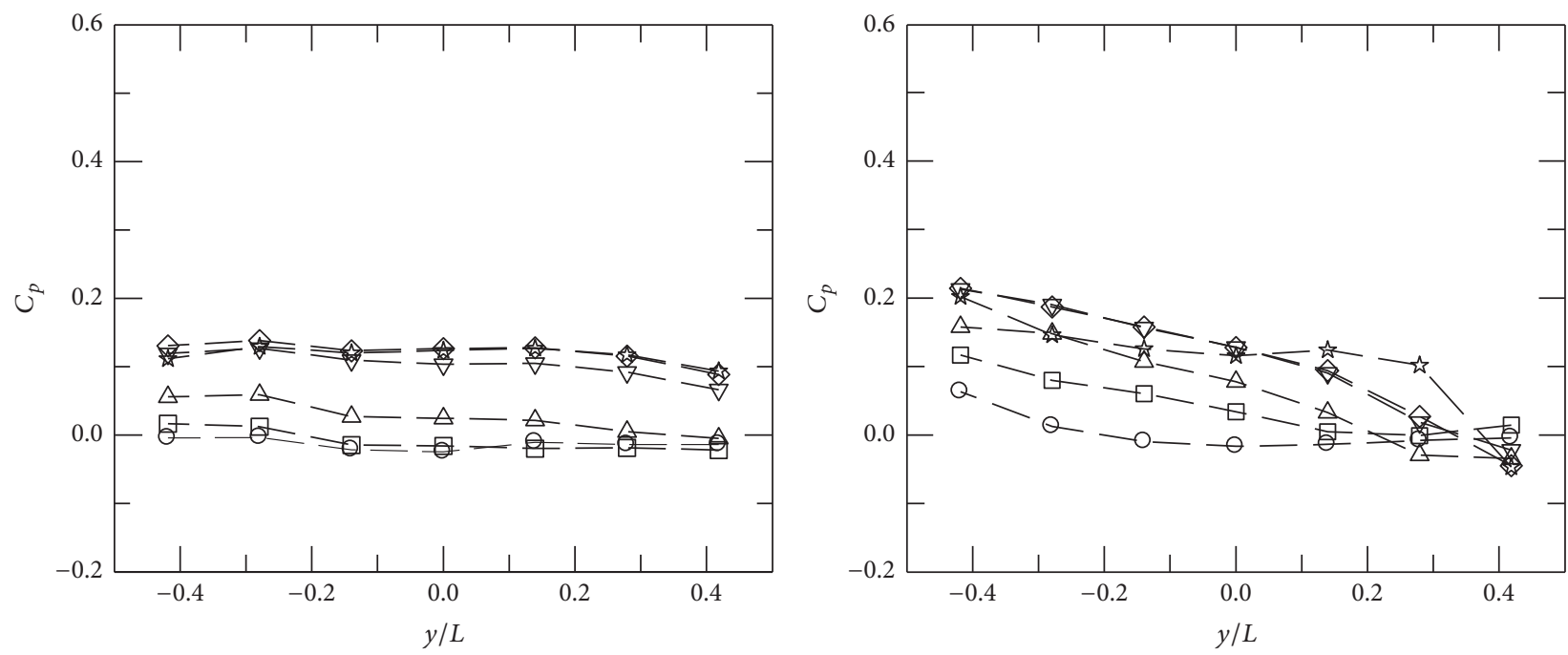

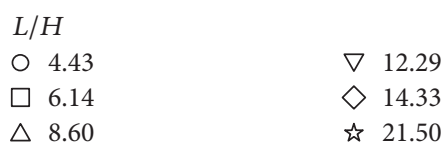

(a) $\beta=10^{\circ}$

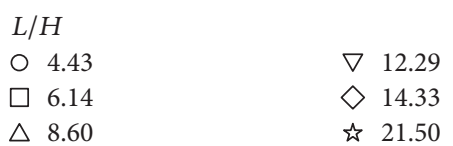

(b) $\beta=45^{\circ}$

FIGURE 9: Span-wise mean surface pressure distributions for $M=0.83$ : (a) $\beta=10^{\circ}$ and (b) $\beta=45^{\circ}$.
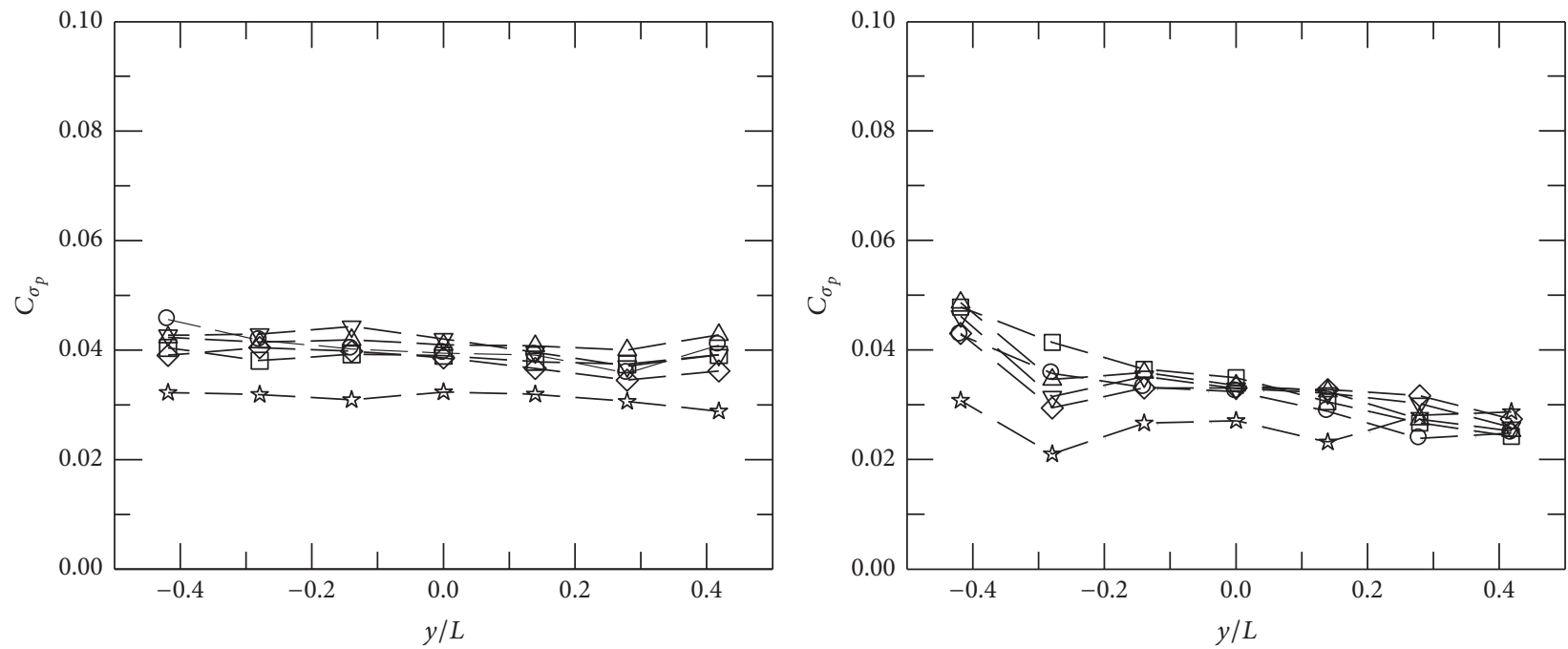

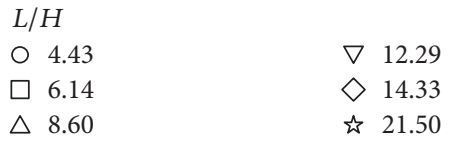

(a) $\beta=10^{\circ}$

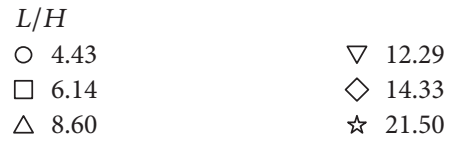

(b) $\beta=45^{\circ}$

FIgURE 10: Span-wise fluctuating pressure distributions for $M=0.83$ : (a) $\beta=10^{\circ}$ and (b) $\beta=45^{\circ}$.

increase in the value of $M$ results in an increase in the value of the SPL.

The variation in $\mathrm{St}_{n}$ with $M$ for $L / H=4.43$ and 6.14 is shown in Figure 13. There is also a prediction using the semiempirical Rossiter's formula $\left(\alpha=0.25\right.$ and $k_{c}=0.57$ for a rectangular cavity). The uncertainty in $\mathrm{St}_{n}$ is estimated to be \pm 0.007 , which is principally due to the resolution of the PSD. It is seen that $\mathrm{St}_{1}=0.26-0.35, \mathrm{St}_{2}=0.60-0.68$, and $\mathrm{St}_{3}=$ $0.97-1.10$. For a given value of $M$, there is a slight decrease in $\mathrm{St}_{2}$ and $\mathrm{St}_{3}$ as $\beta$ increases but not in $S t_{1}$. The empirical 


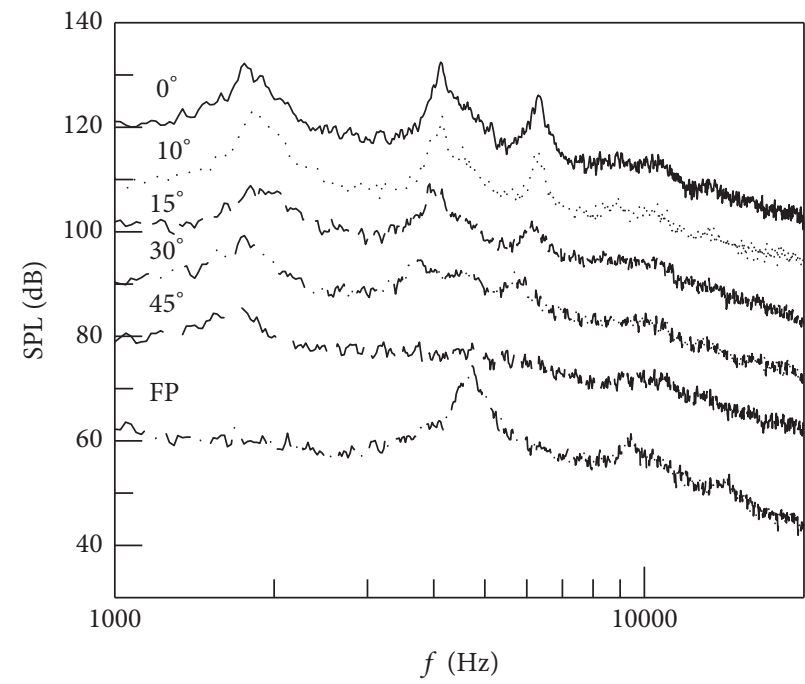

(a) $L / H=4.43$

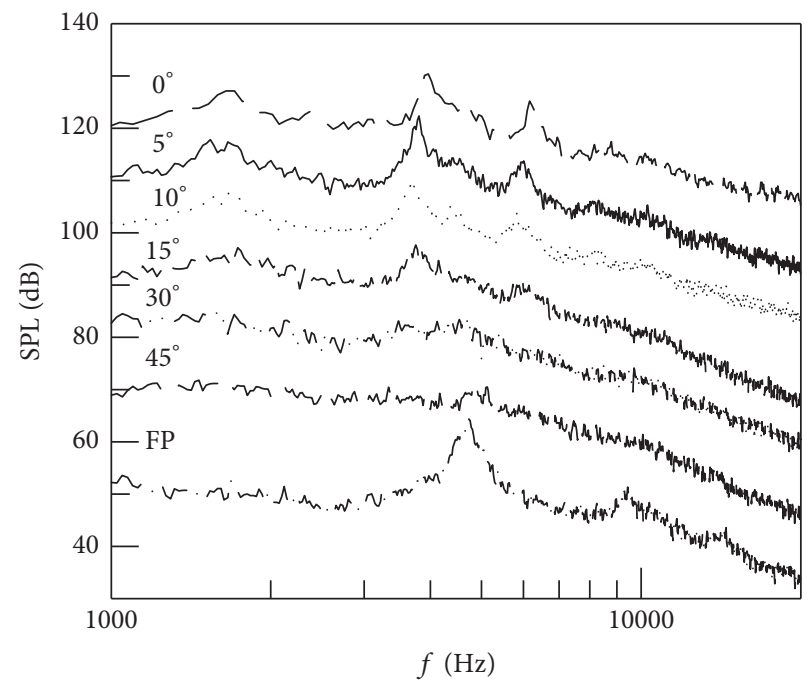

(b) $L / H=6.14$

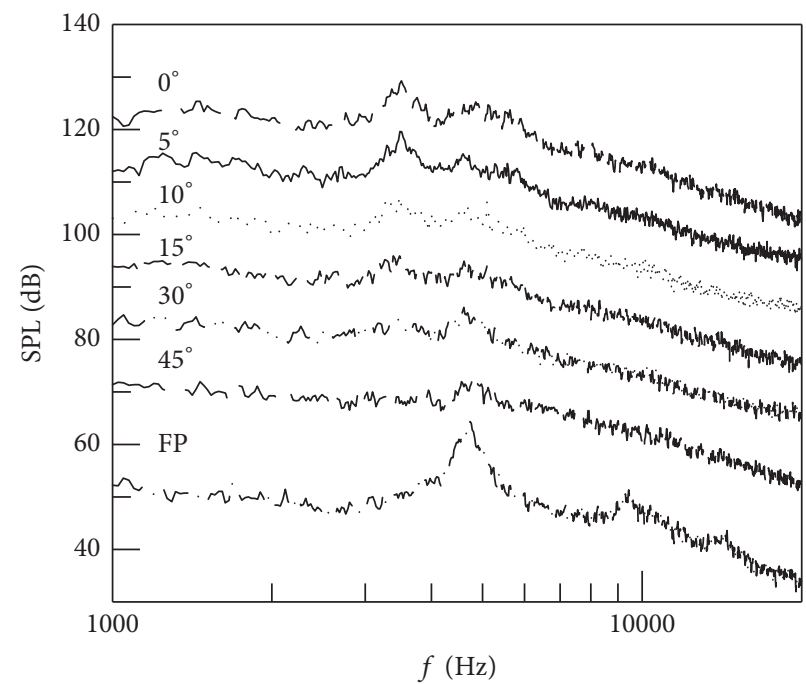

(c) $L / H=8.60$

FIGURE 11: Distributions of the power spectral density for $M=0.83$ and $x / L=0.919$. (Each plot is offset by $10 \mathrm{~dB}$ along the amplitude axis to give greater clarity. The uppermost plot has its original values.)

constants for cavities at yaw are evaluated using the steepest descent method. The optimized values for $\alpha$ and $k_{c}$ are 0.15 and 0.48 , respectively. This demonstrates that a cavity at yaw has a smaller phase lag and a lesser convection velocity.

\section{Conclusions}

This experimental study determines the characteristics of a compressible, yawed rectangular cavity flow. The boundaries for the flow type correspond to $L / H$ and the effect of the value of $\beta$ is smaller. The mean surface pressure gradient in the chord-wise direction at the rear face decreases as the value of $\beta$ increases. The peak amplitude of the fluctuating pressure is significantly less for a large value of $\beta$ for open and transitional cavities. In the span-wise direction, there are asymmetric distributions for $C_{p}$ and $C_{\sigma_{p}}$. These variations are relatively small, compared to those in the chord-wise direction. The resonant frequencies for an open cavity vary slightly with the value of $\beta$ and there is a decrease in the amplitude of the PSD as $\beta$ increases. The resonant modes disappear and the dominant mode changes for large values of $\beta$. Compared to the prediction using Rossiter's semiempirical formula, a cavity at yaw has less lag time and a smaller convection velocity.

\section{Nomenclature}

$C_{p}:$ Static surface pressure coefficient, $\left(p_{w}-q_{\infty}\right) / q_{\infty}$

$C_{\sigma p}$ : Fluctuating pressure coefficient, $\left(\sigma_{p}-\sigma_{p \infty}\right) / q_{\infty}$

$F$ : Objective function

$f_{n}$ : Resonant frequency at mode $n, \mathrm{~Hz}$ 


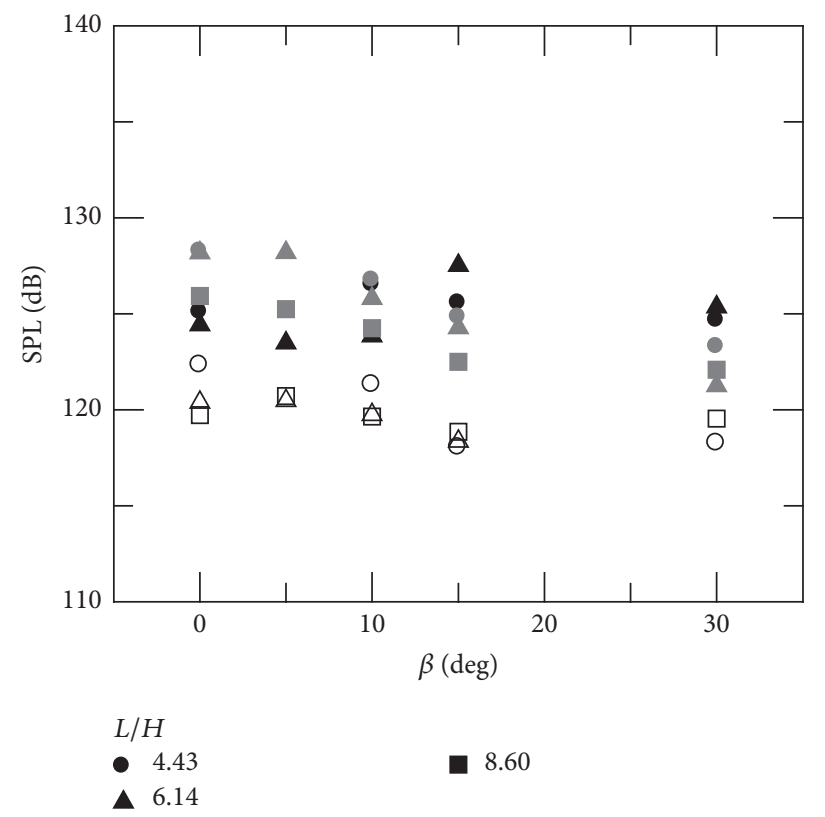

(a) $M=0.64$

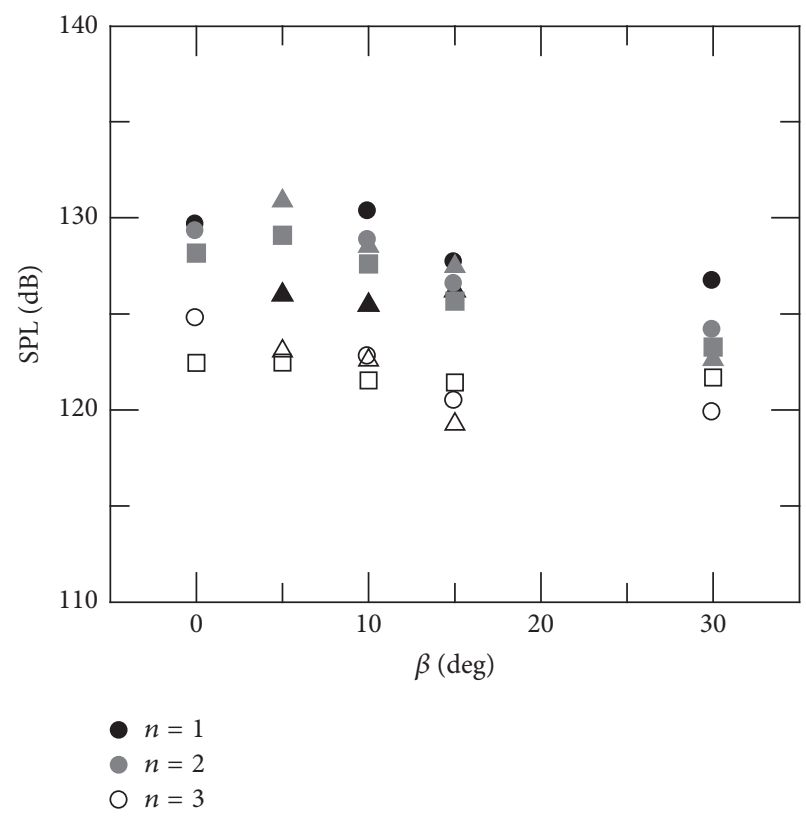

(b) $M=0.70$

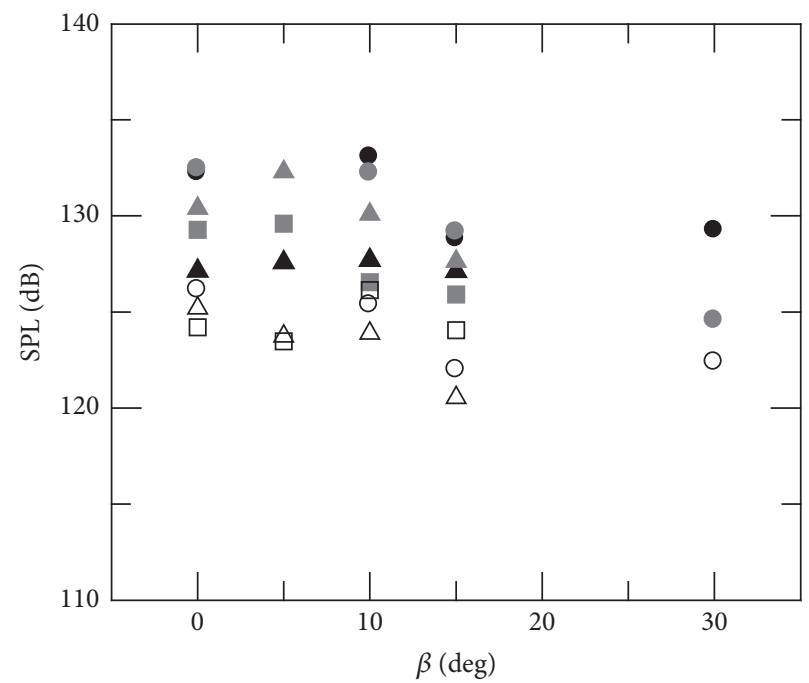

(c) $M=0.83$

FIgURE 12: Amplitude of the resonant modes: (a) $M=0.64$, (b) $M=0.70$, and (c) $M=0.83$.

$k_{c}: \quad$ Ratio of the convection velocity of vortices to freestream velocity

$H$ : $\quad$ Cavity depth

$L: \quad$ Cavity length

M: $\quad$ Freestream Mach number

$N$ : Number of test cases

$n$ : $\quad$ Mode number

PSD: Power spectral density

$p_{w}$ : Static surface pressure

$q_{\infty}$ : Freestream dynamic pressure

SPL: Sound pressure level, $\mathrm{dB}$

$\mathrm{St}_{n}:$ Strouhal number at mode $n, f_{n} L / U_{\infty}$
$U_{\infty}:$ Freestream velocity, $\mathrm{m} / \mathrm{s}$

$W$ : Cavity width

$x$ : Chord-wise distance

$y$ : Span-wise distance

$\alpha: \quad$ Lag time

$\beta$ : $\quad$ Yaw angle, degree

$\delta$ : $\quad$ Boundary thickness

$\sigma_{p}:$ Standard deviation for the surface pressure signal.

\section{Conflicts of Interest}

The authors declare that they have no conflicts of interest. 


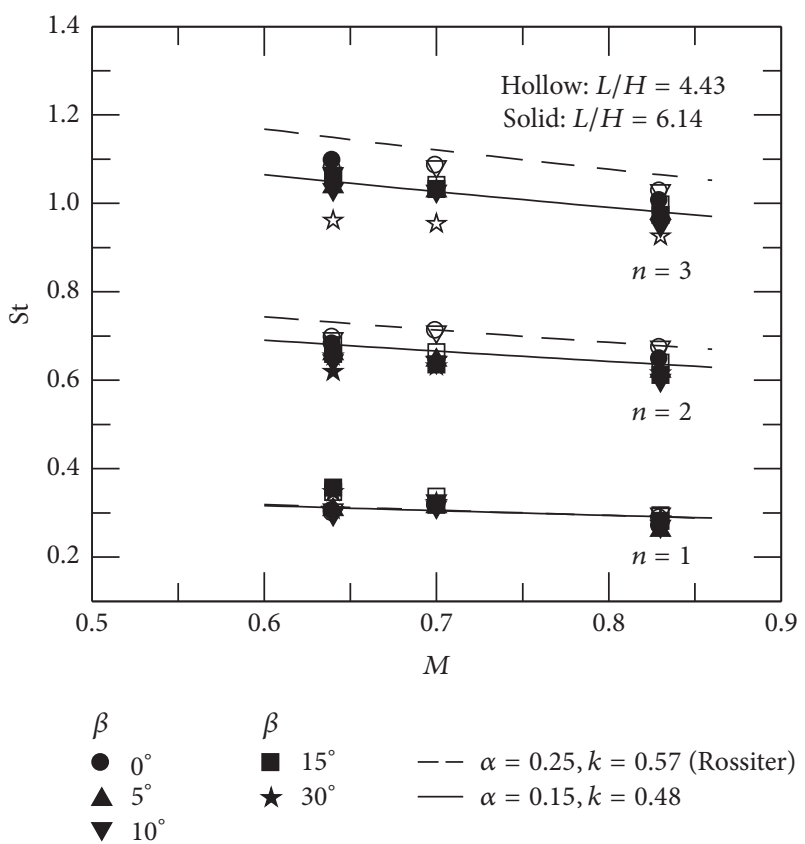

FIGURE 13: The effect of Mach number on the Strouhal number.

\section{Acknowledgments}

The authors acknowledge the support of Ministry of Science and Technology (MOST 104-2926-E-006-006-MY3), Taiwan.

\section{References}

[1] W. Li, "Suppression of supersonic cavity oscillations using pulsed upstream mass injection," International Journal of Aerospace Engineering, vol. 2016, Article ID 6702385, 6 pages, 2016.

[2] T. Handa, K. Tanigawa, Y. Kihara, H. Miyachi, and H. Kakuno, "Frequencies of transverse and longitudinal oscillations in supersonic cavity flows," International Journal of Aerospace Engineering, vol. 2015, Article ID 751029, 2015.

[3] M. B. Tracy and E. B. Plentovich, "Cavity unsteady-pressure measurements at subsonic and transonic speeds," NASA Technical Paper 3669, 1997.

[4] K.-M. Chung, "Characteristics of transonic rectangular cavity flows," Journal of Aircraft, vol. 37, no. 3, pp. 463-468, 2000.

[5] K.-M. Chung, "Three-dimensional effect on transonic rectangular cavity flows," Experiments in Fluids, vol. 30, no. 5, pp. 531536, 2001.

[6] J. Rossiter, "Wind tunnel experiments on the flow over rectangular cavities at subsonic and transonic speeds," Royal Aircraft Establishment, Technical Report 64037, 1964.

[7] D. Rockwell and E. Naudascher, "Review-self-sustaining oscillations of flow past cavities," Journal of Fluids Engineering, vol. 100, no. 2, pp. 152-165, 1978.

[8] E. Savory, N. Toy, P. J. Disimile, and R. G. Dimicco, “The drag of three-dimensional rectangular cavities," Applied Scientific Research, vol. 50, no. 3-4, pp. 325-346, 1993.

[9] M. Czech, E. Savory, N. Toy, and T. Mavrides, "Flow regimes associated with yawed rectangular cavities," Aeronautical Journal, vol. 105, no. 1045, pp. 125-134, 2001.
[10] S. L. Gai, T. J. Soper, and J. F. Milthorpe, "Shallow rectangular cavities at low speeds including effects of yaw," Journal of Aircraft, vol. 45, no. 6, pp. 2145-2150, 2008.

[11] A. Bari and F. Chambers, "Shear layer resonance over open cavities at angles to the flow direction," in 15th Aeroacoustics Conference, Long Beach, Calif, USA.

[12] B. H. K. Lee, D. M. Orchard, and F. C. Tang, "Flow past a yawed rectangular cavity in transonic and low supersonic flows," Journal of Aircraft, vol. 46, no. 5, pp. 1577-1583, 2009.

[13] P. J. Disimile, N. Toy, and E. Savory, "Pressure oscillations in a subsonic cavity at yaw," AIAA Journal, vol. 36, no. 7, pp. 11411148, 1998.

[14] P. J. Disimile and P. D. Orkwis, "Sound-pressure-level variations in a supersonic rectangular cavity at yaw," Journal of Propulsion and Power, vol. 14, no. 3, pp. 392-398, 1998.

[15] K.-M. Chung, P.-H. Chang, and K.-C. Chang, "Tunnel background noise on compressible convex-corner flows," Journal of Aircraft, vol. 50, no. 4, pp. 1011-1015, 2013.

[16] J. S. Bendat and A. G. Piersol, Random Data: Analysis and Measurement Procedures, John \& Wileys, New York, NY, USA, 1986.

[17] K. Chung, "Characteristics of compressible rectangular cavity flows," Journal of Aircraft, vol. 40, no. 1, pp. 137-142, 2003.

[18] Ö. H. Ünalmis, N. T. Clemens, and D. S. Dolling, "Cavity oscillation mechanisms in high-speed flows," AIAA Journal, vol. 42, no. 10, pp. 2035-2041, 2004.

[19] J. A. Snyman, Practical Mathematical Optimization: An Introduction to Basic Optimization Theory and Classical and New Gradient-Based Algorithms, vol. 97 of Applied Optimization, Springer, New York, NY, USA, 2005.

[20] K. H. Lee, Self-Sustained Oscillations of Compressible Cavity Flows [Ph.D. dissertation], National Cheng Kung University, Tainan, Taiwan, 2017.

[21] H. Heller and D. Bliss, "The physical mechanism of flowinduced pressure fluctuations in cavities and concepts for their suppression," in Proceedings of the 2nd Aeroacoustics Conference, Hampton, Va, USA.

[22] A. J. Bilanin and E. Covert, "Estimation of possible excitation frequencies for shallow rectangular cavities," AIAA Journal, vol. 11, no. 3, pp. 347-351, 1973. 


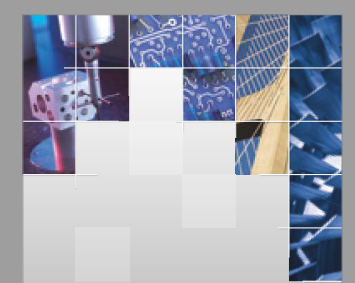

\section{Enfincering}
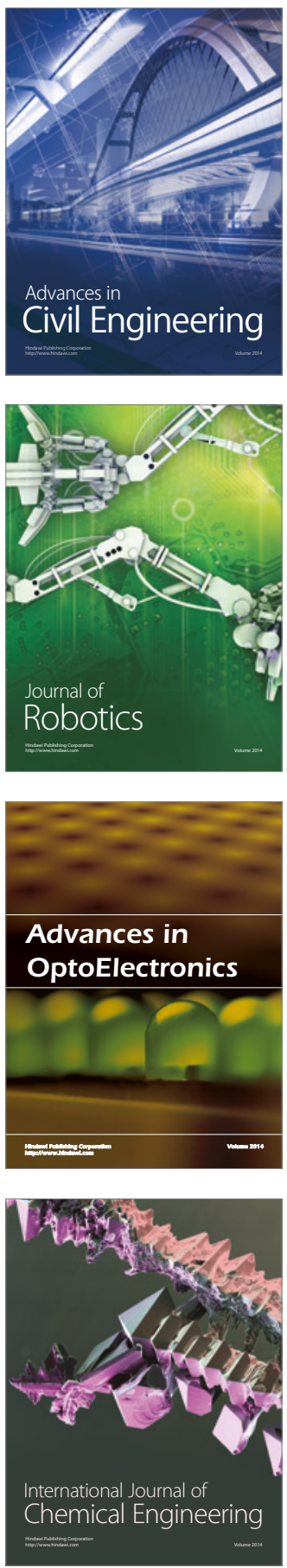

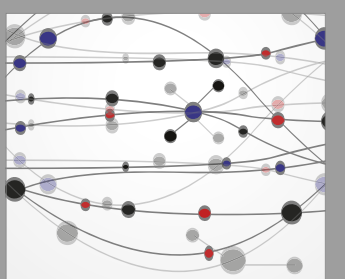

The Scientific World Journal

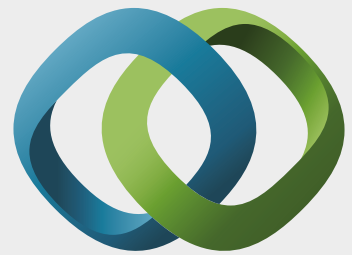

\section{Hindawi}

Submit your manuscripts at

https://www.hindawi.com
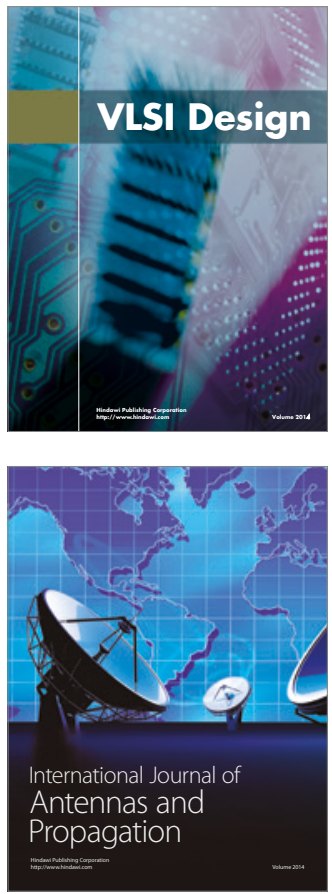

\section{Rotating}

Machinery
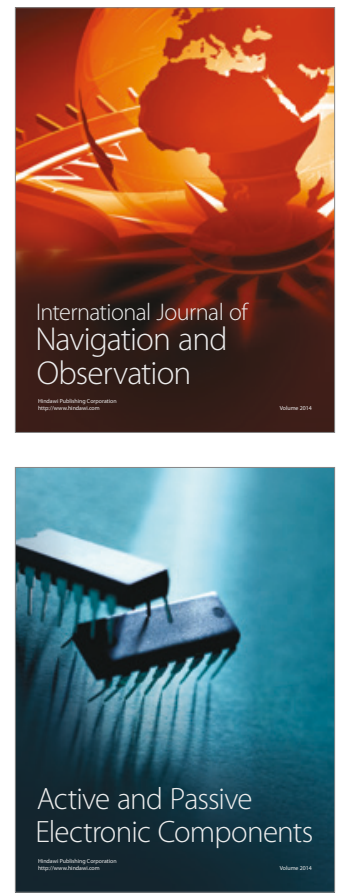
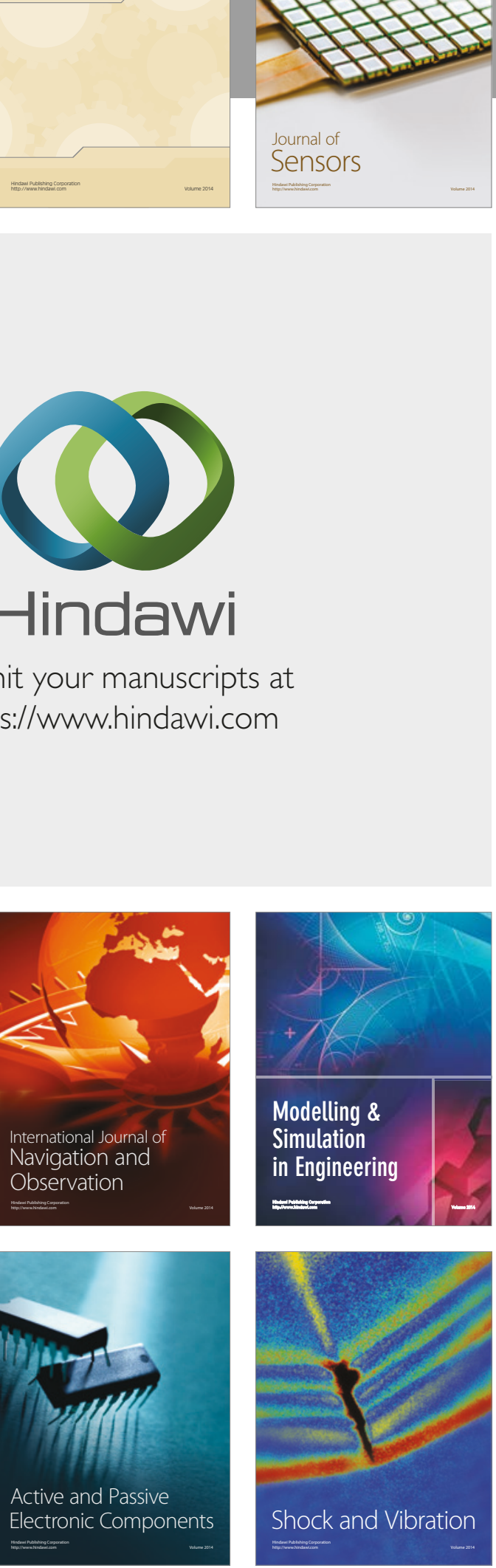
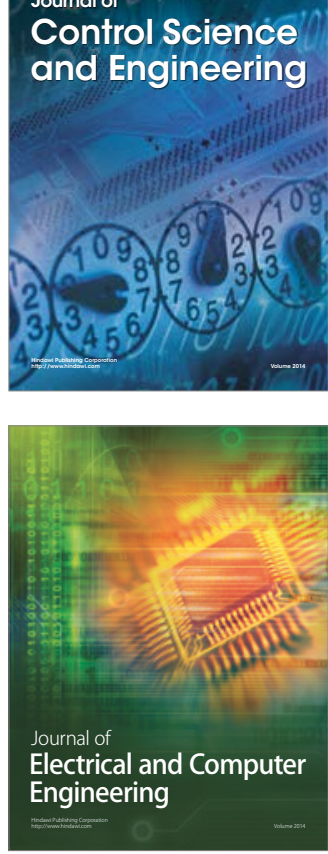

Distributed

Journal of

Control Science

and Engineering
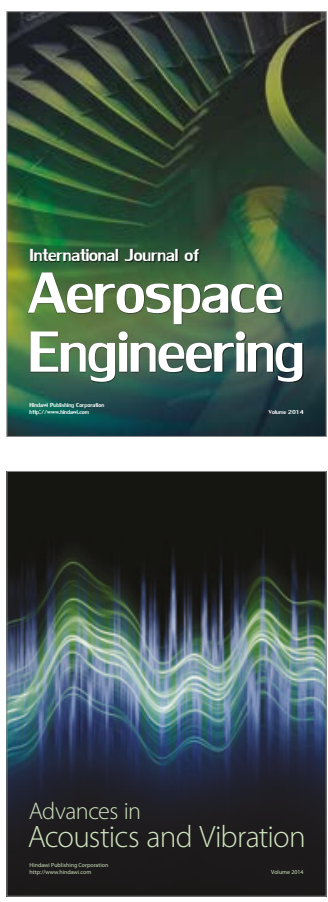

Sensor Networks 